\title{
Non-radiative Solutions of Einstein's Equations for Dust
}

\author{
W. B. Bonnor \\ Department of Mathematics, Queen Elizabeth College, University of London, \\ London W8 7AH, England
}

\begin{abstract}
The quasi-spherical collapsing space-time of Szekeres is investigated. The arbitrary functions can be chosen so that it has positive density, and no Killing vectors; yet a ball $r<r_{0}$ of it can joined to the Schwarzschild metric, and is therefore non-radiative.
\end{abstract}

\section{Introduction}

Whether freely falling bodies radiate gravitational waves is an old question, dating back to the famous work of Einstein, Infeld and Hoffmann on motion. (For a review of early work see [1].) This question has certainly not yet been answered, as has recently been emphasised by Ehlers et al. [2]. Although several approximate methods have shown convincingly that in certain motions of bodies gravitational waves are produced, these methods do not apply to free fall; and approximation methods specially designed for free fall have not yet produced satisfactory results [2].

An answer could be provided by appropriate exact solutions of Einstein's equations for dust, which is a good model for freely falling matter. Until 1975 the known exact solutions for dust, all of which had high spatial symmetry, seemed, with one possible exception, not to contain gravitational radiation, though it is hard to test whether radiation is present or not. The exception was the case of cylindrical symmetry for which Cocke [3] claimed to find an energy transmission corresponding to gravitational waves. I am not convinced by this work for two reasons. First, the concept of energy in the cylindrical case has not been satisfactorily formulated, partly because the space-time is not Minkowskian at infinity; secondly, since Cocke's cylinders are infinite in length, some interference with the energy balance by an influx at the ends cannot be ruled out. Thus we can say that exact solutions up to 1975 gave an inconclusive answer to our question.

In 1975 Szekeres $[4,5]$ published an exact solution which is much more promising, and which will be investigated in this paper. The basic idea of this work is to show that a portion of the solution having finite spatial volume can be joined to a Schwarzschild metric. Since the latter is static, it follows that, whatever 
may be going on inside the core, it is not radiating to the outside world, and is therefore non-radiative. Two subsidiary results are needed to make the conclusion interesting: first, that this can be done with a mass density everywhere bounded and non-negative; secondly, that Szekeres's solution has in general no Killing vectors. The second point, which will be proved in another paper, is important because it shows that free fall may be non-radiative even when unsymmetric, and also because it proves that Szekeres's solution is distinct from previously known solutions, which do have Killing vectors.

In Sections 2 and 3 the solution is given and interpreted. Section 4 contains the main result of the paper in the form of two matching theorems. In Section 5 I show that the physical requirement of positive, bounded density can be met, and in Section 6 there is an example of a Szekeres space-time joined to a Schwarzschild metric. The paper ends with a conclusion.

\section{Szekeres's Quasi-spherical Collapsing Space-Time}

The metric is

$$
d s^{2}=-e^{\lambda} d r^{2}-e^{\omega}\left(d y^{2}+d z^{2}\right)+d t^{2},
$$

where $\lambda, \omega$ are functions of $r, y, z$, and $t$. The field equations are those for dust

$$
R^{i k}-\frac{1}{2} g^{i k} R=-8 \pi \varrho u^{i} u^{k},
$$

where $R^{i k}$ is the Ricci tensor, $\varrho$ the density and $u^{i}$ the unit four-velocity, given by $u^{i}=\delta_{4}^{i}$ because the coordinates are comoving. Szekeres found two classes of solutions, and the class we are interested in here is that of quasi-spherical collapsing space-times, though by simple modifications it can refer to expanion from singularities. It is given by

$$
\begin{aligned}
& e^{\omega / 2}=\phi(r, t)[P(r, y, z)]^{-1}, \quad e^{\lambda / 2}=P[W(r)]^{-1} \frac{\partial}{\partial r}\left(e^{\omega / 2}\right), \\
& P=a(r)\left(y^{2}+z^{2}\right)+2 f(r) y+2 g(r) z+c(r), \\
& a c-f^{2}-g^{2}=\frac{1}{4}
\end{aligned}
$$

$\phi$ is a Friedmann function, that is, it satisfies the Friedmann equation

$$
\phi_{4}^{2}=W^{2}-1+\phi^{-1} S(r) .
$$

Throughout the paper suffixes $1,2,3,4$ denote differentiation with respect to $r$, $y, z, t$ respectively. The solution contains so far six functions of integration which depend on $r$ only. A seventh arises when one integrates (2.6). Writing (2.6) as

$$
\left(W^{2}-1+\phi^{-1} S\right)^{-1 / 2} d \phi=d t
$$

we can set down its formal solution as

$$
\int\left(W^{2}-1+\phi^{-1} S\right)^{-1 / 2} d \phi=t+H(r),
$$


$H$ being the seventh function of integration. Of the seven functions, $a, f, g, c, W$, $S, H$, five are independent; this is because there is one relation (2.5) between them, and, further, one function of $r$ can be given an assigned form by a transformation of coordinates $r^{\prime}=F(r)$. Hence the solution contains five independent arbitrary functions of one variable $r$. I shall suppose all functions of integration to be of class $C^{3}$, except at surfaces of discontinuity, which will be discussed in Section 4 . The density is given by

$$
8 \pi \varrho=\left(P S_{1}-3 S P_{1}\right) \phi^{-2}\left(P \phi_{1}-\phi P_{1}\right)^{-1} .
$$

Let us assume that initially at $t=0$ the metric is non-singular, and that all parts of the dust cloud are collapsing:

$$
\phi_{4}(r, 0) \leqq 0 .
$$

The ranges of the coordinates will be taken as

$$
0 \leqq d_{1} \leqq r \leqq d_{2}, \quad-\infty<y, z<\infty, \quad 0 \leqq t<t_{c},
$$

where $d_{1}, d_{2}$ are constants, where

$$
t_{c}=\min \left(t_{0}, t_{*}\right)
$$

and

$$
\begin{aligned}
& \phi\left(r, t_{0}\right)=0, \quad\left(t_{0}>0\right), \\
& P(r, y, z) \phi_{1}\left(r, t_{*}\right)-\phi\left(r, t_{*}\right) P_{1}(r, y, z)=0, \quad\left(t_{*}>0\right) .
\end{aligned}
$$

The meaning of (2.10)-(2.13) is as follows: the particles of the collapsing dust cloud may reach a singularity through (2.12) or (2.13) [5]. We wish the solution to apply up to whichever singularity occurs first after $t=0$, and this is ensured by $t<t_{c}$ in (2.10). Consequently we shall henceforth assume that in the domain of validity of our solution

$$
\phi \neq 0, \quad P \phi_{1}-\phi P_{1} \neq 0 .
$$

(We ignore a trivial coordinate singularity $\phi(0, t)=0$.) Moreover it is clear from (2.5) that we need

$$
a \neq 0
$$

from which it follows, since

$$
P=a\left[\left(y+f a^{-1}\right)^{2}+\left(z+g a^{-1}\right)^{2}+(2 a)^{-2}\right]
$$

that

$$
P \neq 0 .
$$

Finally we suppose that

$$
W \neq 0 \text {. }
$$

The solution described in this section will be called Szekeres's quasi-spherical collapsing space-time. 


\section{Interpretation}

Consider the 2-surfaces $\Sigma: r=r_{1}$ (const), $t=t_{1}$ (const). Because $a, f, g$ in (2.16) are constant on $\Sigma$, the metric on $\Sigma$ can by a transformation of the form

$$
y^{\prime}=e y+k_{1}, \quad z^{\prime}=e z+k_{2}, \quad\left(e, k_{1}, k_{2} \text { const }\right)
$$

be brought to the form

$$
d \sigma^{2}=\varphi_{*}^{2}\left[d y^{\prime 2}+d z^{\prime 2}\right]\left[\frac{1}{2}\left(1+y^{\prime 2}+z^{\prime 2}\right)\right]^{-2}, \quad \phi_{*}=\phi\left(r_{1} \cdot t_{1}\right) .
$$

The additional transformation

$$
y^{\prime}+i z^{\prime}=\cot \frac{1}{2} \theta e^{i \alpha}
$$

takes the metric of $\Sigma$ into

$$
d \sigma^{2}=\phi_{*}^{2}\left(d \theta^{2}+\sin ^{2} \theta d \alpha^{2}\right) .
$$

Hence $\Sigma$ are spheres of radius $\phi_{*}$, and $y$ and $z$ are stereographic coordinates. However, for different values of $r$, the point in the $y-z$ plane representing the centres of the spheres, given by $\left(-k_{1} / e,-k_{2} / e\right)$, will be different. Moreover, if $r$ is not constant it is not possible to eliminate $a$. For these reasons we may call the geometry quasi-spherical. However, for general values of the arbitrary functions it certainly is not spherically symmetric; indeed it has no Killing vectors at all [6].

From (2.8) we find that the density depends on $y$ and $z$ unless either

$$
a_{1} / a=f_{1} / f=g_{1} / g=c_{1} / c
$$

which leads to spherical symmetry; or

$$
S_{1} / 3 S=\phi_{1} / \phi
$$

in which case $\phi$ is a product of a function of $r$ and a function of $t$, so that (2.1) reduces to

$$
d s^{2}=-F(t) h_{\alpha \beta} d x^{\alpha} d x^{\beta}+d t^{2},
$$

where $\alpha, \beta=1,2,3$ and $h_{\alpha \beta}$ is independent of $t$. It is now possible to invoke a theorem of Szekeres [4] to show that the space-time is a homogeneous Friedmann cosmology.

The dynamical properties of the model in Section 2 are governed by (2.6), which is the same equation as one gets in spherically symmetric collapse of dust [7]. It reduces to the equation of homogeneous dust cosmologies if we put

$$
W^{2}=1+\varepsilon r^{2}, \quad S=K r^{3},
$$

where $\varepsilon=0, \pm 1$ and $K$ is a constant. (2.6) has solutions of the three well-known

$$
=1
$$

types-parabolic, elliptic or hyperbolic-according as $W^{2}<1$.

$$
>1
$$

\section{Matching}

I shall now prove the main results of the paper.

Theorem 1. Let $r_{0}$ be a positive constant, and let $V$ denote a Szekeres quasispherical collapsing space-time with domain

$$
r_{0}<r, \quad-\infty<y, z<\infty, \quad 0 \leqq t<t_{c},
$$


and functions of integration $a, f, g, c, S, W, H$; let $\bar{V}$ denote another such spacetime with domain

$$
0 \leqq \bar{r}<r_{0}, \quad-\infty<\bar{y}, \bar{z}<\infty, \quad 0 \leqq \bar{t}<\bar{t}_{c},
$$

and functions of integration $\bar{a}, \bar{f}, \bar{g}, \bar{c}, \bar{S}, \bar{W}, \bar{H}$; let $B$ be the boundary between $V$ and $\bar{V}$, denoted by $r=r_{0}$ in $V$, and $\bar{r}=r_{0}$ in $\bar{V}$. Then $V$ and $\bar{V}$ match across $S$ if

$$
S\left(r_{0}\right)=\bar{S}\left(r_{0}\right), \quad W\left(r_{0}\right)=\bar{W}\left(r_{0}\right), \quad H\left(r_{0}\right)=\bar{H}\left(r_{0}\right) .
$$

Proof. We first note that since $r$ is constant on $B$ we may use the transformation theory of Section 3 to simplify the metric which $B$ inherits from $V$. Let us carry out on $V$ the transformation (3.1) with constants $e, k_{1}, k_{2}$ chosen so that on $B$ the metric of $V$ reduces to

$$
d S^{2}=-\left[\phi\left(r_{0}, t\right)\right]^{2}\left[\frac{1}{2}\left(1+y^{\prime 2}+z^{\prime 2}\right)\right]^{-2}\left(d y^{\prime 2}+d z^{\prime 2}\right)+d t^{2} .
$$

Elsewhere in $V$ the metric will still have the form (2.1), plus (2.3)-(2.5). In a similar way we reduce the metric which $B$ inherits from $\bar{V}$ to

$$
d \bar{S}^{2}=-\left[\bar{\phi}\left(r_{0}, \bar{t}\right)\right]^{2}\left[\frac{1}{2}\left(1+\bar{y}^{2}+\bar{z}^{\prime 2}\right)\right]^{-2}\left(d \bar{y}^{\prime 2}+d \bar{z}^{\prime 2}\right)+d \bar{t}^{2} .
$$

Henceforth we suppose this done in both $V$ and $\bar{V}$, and drop the primes.

For matching we must ensure that the first fundamental form of $B$ is the same whether it is calculated from the metric of $V$ or from that of $\bar{V}$; and likewise for the second fundamental form. Let $B$ have representations in $V, \bar{V}$ :

$$
\begin{aligned}
& \text { in } \left.V, \quad x^{1} \equiv r=r_{0}, \quad x^{2} \equiv y=u^{2}, \quad x^{3} \equiv z=u^{3}, \quad x^{4} \equiv t=u^{4} ;\right\} \\
& \text { in } \left.\bar{V}, \quad \bar{x}^{1} \equiv \bar{r}=r_{0}, \quad \bar{x}^{2} \equiv \bar{y}=u^{2}, \quad \bar{x}^{3} \equiv \bar{z}=u^{3}, \quad \bar{x}^{4} \equiv \bar{t}=u^{4} ;\right\}
\end{aligned}
$$

so that we identify points on both sides of $B$ according to

$$
y=\bar{y}, \quad z=\bar{z}, \quad t=\bar{t} .
$$

Substituting (4.4) in (4.2) and (4.3) (without the primes) we see that identity on both sides of $B$ of the first fundamental forms follows if

$$
\phi\left(r_{0}, u^{4}\right)=\bar{\phi}\left(r_{0}, u^{4}\right)
$$

which will be so if (4.1) is satisfied.

The coefficients of the second fundamental form of $B$, regarded as a hypersurface in $V$, are given by

$$
\Omega_{\mu v}=-n_{a ; b} \frac{\partial x^{a}}{\partial u^{\mu}} \frac{\partial x^{b}}{\partial u^{v}}
$$

where $a, b=1,2,3,4$ and $\mu, v=2,3,4 ; n_{a}$ is the unit normal to $B$ and; denotes covariant differentiation with respect to the four dimensional space; $x^{a}$ are the coordinates referred to in (4.4). The normal is calculated as follows: $B$ may be written as

$$
f\left(x^{i}\right) \equiv x^{1}-r_{0}=0
$$

regarded as a hypersurface in $V$. Then the normal is 
where , means partial differentation, and we easily find

$$
n_{a}=\delta_{a}^{1} e^{\lambda / 2} .
$$

An exactly similar definition applies to $\bar{\Omega}_{\mu v}$, the second fundamental form on $B$, regarded as a surface in $\bar{V}$.

When we calculate $\Omega_{\mu v}$ we find that derivatives with respect to $r$ cancel; in fact the non-zero components of $\Omega_{\mu v}$ are

$$
\Omega_{22}=\Omega_{33}=\Gamma_{22}^{1} n_{1} \ldots-\left[\frac{W \phi}{P^{2}}\right]_{r=r_{0}}=-W\left(r_{0}\right) \phi\left(r_{0}, u^{4}\right)\left[\frac{1}{2}\left(1+y^{2}+z^{2}\right)\right]^{-2},
$$

and similar expressions for $\bar{\Omega}_{\mu v}$. Then $\Omega_{\mu v}=\bar{\Omega}_{\mu v}$.

Hence, given (4.1), both first and second fundamental forms of $B$ have the required properties, and the theorem is proved.

Q.E.D.

Theorem 2. Every Szekeres space-time $\bar{V}$ defined in Theorem 1 can be matched to a Schwarzschild vacuum space-time (denoted by E).

Proof. Let $\bar{V}$ be a Szekeres space-time in which, as in Theorem 1, coordinates $\bar{y}, \bar{z}$ have been chosen so that

$$
\bar{a}\left(r_{0}\right)=\bar{c}\left(r_{0}\right)=\frac{1}{2}, \quad \bar{f}\left(r_{0}\right)=\bar{g}\left(r_{0}\right)=0 .
$$

Let $V$, the matching space-time of Theorem 1 be specialised so that

$$
\left.\begin{array}{l}
a(r)=c(r)=\frac{1}{2}, \quad f(r)=g(r)=0, \\
S(r)=\bar{S}\left(r_{0}\right), \quad W\left(r_{0}\right)=\bar{W}\left(r_{0}\right), \quad H\left(r_{0}\right)=\bar{H}\left(r_{0}\right) .
\end{array}\right\}
$$

Note that in the metric of $V$ all arbitrary functions except $W$ and $H$ are constants and that $\partial P / \partial r=0, d S / d r=0$. It follows from (2.8) that $\varrho=0$, so this is a vacuum space-time. Moreover its metric can be reduced to

$$
d s^{2}=-\left(\phi_{1} W^{-1}\right)^{2} d r^{2}-\phi^{2}\left(d \theta^{2}+\sin ^{2} \theta d \alpha^{2}\right)+d t^{2}
$$

by a transformation of form (3.3). Hence it is spherically symmetric, and so by Birkhoff's theorem it must be an $E$.

Further, it is clear that this $E$ matches $\bar{V}$ since by (4.9) the conditions of Theorem 1 are fulfilled.

Q.E.D.

Dr. Geroch has pointed out to me that a slightly weaker version of this theorem is obvious from the fact that Szekeres's solutions (2.1) contain the Schwarzschild solution as a special case. Take any functions $a, c, f, g, S, W, H$ which satisfy (4.9) for $r>r_{0}$, and continue them inside $r<r_{0}$ in such a way that they satisfy (2.5) and also are of class $C^{3}$ (in accordance with our assumption of Section 2 that all functions of integration shall be so) at $r=r_{0}$. Then the whole constitutes a Szekeres solution covered by one chart; it can contain inhomogeneous dust inside $r=r_{0}$, and is Schwarzschild outside. This theorem is weaker than Theorem 2 only because the arbitrary functions have to be of class $C^{3}$ at the boundary.

\section{Conditions for Positive, Finite Density}

We are interested only in solutions with non-negative and finite matter density $\varrho$, and in this Section I show that these requirements can be ensured by mild restrictions on the arbitrary functions. 
In Section 2 we assumed that at $t=0$ the metric is non-singular, and the spacetime collapsing. We make the further assumption that $\phi(r, 0)$ is monotonically increasing, so that by a coordinate transformation of $r$ it can be brought to the form

$$
\phi(r, 0)=r .
$$

We strengthen assumption (2.15) to

$$
a>0 \text {. }
$$

Under these assumptions it was shown by Szekeres [5] that

$$
P \phi_{1}(r, 0)-P_{1} \phi(r, 0)>0
$$

if

$$
a_{1} c_{1}-f_{1}^{2}-g_{1}^{2}>-(2 r)^{-2}
$$

and that

$$
P S_{1}-3 P_{1} S \geqq 0
$$

if

$$
a_{1} c_{1}-f_{1}^{2}-g_{1}^{2}>-\left(S_{1} / 6 S\right)^{2}
$$

and

$$
L t_{r \rightarrow 0} r^{-3} S=K \text { (const) }, \quad(K>0) .
$$

From (2.8) we see that with the foregoing assumptions $\varrho$ is positive and finite on $t=0$. Then by assumptions (2.14) no infinities or sign changes can occur in $\varrho$ during the time interval $0 \leqq t<t_{c}$.

To sum up, $\varrho$ is finite and non-negative in the domain of our model provided assumptions (5.1), (5.2), (5.4), (5.6), and (5.7) are satisfied. These constraints on the arbitrary functions are very mild, and do not seriously restrict the set of solutions.

\section{An Example}

I illustrate the foregoing by the example of a parabolic, or zero-energy, collapse: that is, I put

$$
W=1
$$

throughout. Equation (2.6) integrates to give

$$
\phi=\left\{H(r)-\frac{3}{2}[S(r)]^{1 / 2} t\right\}^{2 / 3},
$$

the positive square root being taken. If we suppose $H$ monotonic, we can by a coordinate change in $r$ reduce this to

$$
\phi=\left\{r^{3 / 2}-\frac{3}{2}[S(r)]^{1 / 2} t\right\}^{2 / 3}
$$

The four functions $a, f, g, c$ of $r$ in (2.4) are arbitrary subject to (2.5), and so is $S$, subject to $S=0\left(r^{3}\right)$ as $r \rightarrow 0$. 
I now construct the space-time $\bar{V}$ matched to a Schwarzschild vacuum spacetime $E$. The complete solution is given by

$$
\begin{aligned}
& \left.\begin{array}{rl}
\bar{V}: 0 \leqq \bar{r}<r_{0} \\
\hline \bar{\phi} & =\left\{\bar{r}^{3 / 2}-\frac{3}{2}[\bar{S}(\bar{r})]^{1 / 2} \bar{t}\right\}^{2 / 3}, \\
\bar{P} & =\bar{a}(\bar{r})\left(\bar{y}^{2}+\bar{z}^{2}\right)+2 \bar{f}(\bar{r}) \bar{y}+2 \bar{g}(\bar{r}) \bar{z}+\bar{c}(\bar{r}),
\end{array}\right\} \\
& \left.\begin{array}{l}
\frac{E: r_{0}<r}{\phi}=\left\{r^{3 / 2}-\frac{3}{2}[2 m]^{1 / 2} t\right\}^{2 / 3}, \quad(m \text { const }) \\
P=\frac{1}{2}\left(y^{2}+z^{2}+1\right) .
\end{array}\right\}
\end{aligned}
$$

The boundary $B$ is $r=\bar{r}=r_{0}$ and the boundary conditions required by (4.1) are

$$
\bar{S}\left(r_{0}\right)=2 m, \quad \bar{a}\left(r_{0}\right)=\bar{c}\left(r_{0}\right)=\frac{1}{2}, \quad \bar{f}\left(r_{0}\right)=\bar{g}\left(r_{0}\right)=0 .
$$

Transforming to spherical polar coordinates by (3.3) we obtain for the metric of $E$

$$
d s^{2}=-r R^{-1} d r^{2}-R^{2}\left(d \theta^{2}+\sin ^{2} \theta d \alpha^{2}\right)+d t^{2},
$$

where

$$
R=\left\{r^{3 / 2}-\frac{3}{2}[2 m]^{1 / 2} t\right\}^{2 / 3} .
$$

(6.7) is transformed to the usual Schwarzschild metric

$$
d s^{2}=-\left(1-\frac{2 m}{R}\right)^{-1} d R^{2}-R^{2}\left(d \theta^{2}+\sin ^{2} \theta d \alpha^{2}\right)+\left(1-\frac{2 m}{R}\right) d \tau^{2}
$$

by (6.8) together with

$$
\tau=-t+2(2 m)^{1 / 2} R^{1 / 2}+2 m\left\{\log \left[R^{1 / 2}-(2 m)^{1 / 2}\right]-\log \left[R^{1 / 2}+(2 m)^{1 / 2}\right]\right\} .
$$

The form (6.7) of $E$ is due to H. P. Robertson.

Thus the interior quasi-spherical collapsing space-time given by (6.4) matches the Schwarzschild vacuum space-time with metric (6.7) or (6.9). The relation $2 m=\bar{S}\left(r_{0}\right)$ is in agreement with the formula for the mass $m\left(r_{0}\right)$ in a comoving volume of radius $r_{0}$ of the space-time (2.1) [5] which is

$$
m\left(r_{0}\right)=\frac{1}{2} \int_{0}^{r_{0}} \bar{S}_{1} \bar{W}^{-1} d \bar{r} .
$$

Putting $\bar{W}=1$ and integrating we have $m\left(r_{0}\right)=\frac{1}{2} \bar{S}\left(r_{0}\right)$ since $\bar{S}(0)=0$.

In this example we have used the metric (6.7) which describes $E$ in freely falling (or comoving) coordinates. In these coordinates the curves

$$
r=\text { const }, \quad \theta=\text { const }, \quad \alpha=\text { const }
$$

are geodesics. Because we put $W=1$ we were led to parabolic (or zero energy) comoving coordinates. If $W \neq 1$ an analogous procedure can still be followed but one needs the Schwarzschild solution in elliptic or hyperbolic comoving coordinates [8]. 


\section{Conclusion}

Any portion $r<r_{0}$ of a Szekeres metric can be matched to an exterior Schwarzschild solution. If we then consider the interior and exterior space-times together we can say that since there is no radiation in the Schwarzschild portion, the Szekeres core is non-radiative. One might object that this does not rule out radiative processes in $r<r_{0}$; my reply would be that a flow of energy away from the system is an essential feature of a radiative process.

Our solution is not complete: it is a slice of finite duration, in accordance with (2.10). However, this does not invalidate the conclusion because if radiation were present it should show up between $t=0$ and $t=t_{c}$.

The surfaces $r=r_{0}$ are spheres, and this work deals only with a ball of unsymmetrically moving dust. A similar result may or may not apply to other shapes carved out of Szekeres's solution.

It would be rash to conjecture from this work that no freely falling matter radiates. The Szekeres solution has a good deal of symmetry, even though it has in general no Killing vectors. It may be that more unsymmetrical motions do radiate. One of the difficulties of investigating this-apart, of course, from finding the solutions!-will be to discover a finer classification of symmetries than is given by the mere presence or absence of Killing vectors.

\section{References}

1. Bonnor, W. B.: Brit. J. Appl. Phys. 14, 555-562 (1963)

2. Ehlers, J., Rosenblum, A., Goldberg, J. N., Havas, P.: Ap. J. 208, L77-L81 (1976)

3. Cocke, W. J.: J. Math. Phys. 7, 1171-1178 (1966)

4. Szekeres, P.: Commun. math. Phys. 41, 55-64 (1975)

5. Szekeres, P.: Phys. Rev. D 12, $2941-2948$ (1975)

6. Bonnor, W.B., Sulaiman,A.H., Tomimura, N.: to be published

7. Tolman, R. C.: Proc. Nat. Acad. Sci. 20, 169-183 (1934)

8. Szekeres, P.: Nuovo Cimento 17 B, 187-195 (1973)

Communicated by R. Geroch

Received August 6, 1976 
